\title{
Molecular mobility, composition and structure analysis in glycerol plasticised chitosan films
}

\author{
Joana F. Fundo ${ }^{\text {a }}$, Rui Fernandes ${ }^{\mathrm{b}}$, Pedro M. Almeida ${ }^{\mathrm{c}, \mathrm{d}}$, Alexandra Carvalho ${ }^{\mathrm{d}}$, Gabriel Feio ${ }^{\mathrm{d}}$, \\ Cristina L.M. Silva ${ }^{\text {a,* }}$, Mafalda A.C. Quintas ${ }^{a, e}$ \\ ${ }^{a}$ CBQF - Centro de Biotecnologia e Química Fina, Escola Superior de Biotecnologia, Centro Regional do Porto da Universidade Católica Portuguesa, Porto, Portugal \\ ${ }^{\mathrm{b}}$ IBMC/HEMS - Instituto de Biologia Molecular e Celular/Histology and Electron Microscopy Service, Universidade do Porto, Porto, Portugal \\ ${ }^{\mathrm{c}}$ ADF - Instituto Superior de Engenharia de Lisboa, IPL, Lisboa, Portugal \\ ${ }^{\mathrm{d}}$ CENIMAT-I3N, Departamento de Ciência dos Materiais, Faculdade de Ciências e Tecnologia, Universidade Nova de Lisboa, Caparica, Portugal \\ e IBB - Institute for Biotechnology and Bioengineering, Centre of Biological Engineering, Universidade do Minho, Braga, Portugal
}

\section{A R T I C L E I N F O}

Article history:

Available online 5 June 2013

\section{Keywords:}

Edible films

Microstructure

NMR

Molecular dynamics

Structure-function

Water behaviour

\begin{abstract}
A B S T R A C T
This study was developed with the purpose to investigate the effect of polysaccharide/plasticiser concentration on the microstructure and molecular dynamics of polymeric film systems, using transmission electron microscope imaging (TEM) and nuclear magnetic resonance (NMR) techniques. Experiments were carried out in chitosan/glycerol films prepared with solutions of different composition. The films obtained after drying and equilibration were characterised in terms of composition, thickness and water activity.

Results show that glycerol quantities used in film forming solutions were responsible for films composition; while polymer/total plasticiser ratio in the solution determined the thickness (and thus structure) of the films. These results were confirmed by TEM.

NMR allowed understanding the films molecular rearrangement. Two different behaviours for the two components analysed, water and glycerol were observed: the first is predominantly moving free in the matrix, while glycerol is mainly bounded to the chitosan chain.
\end{abstract}

(c) 2013 Elsevier Ltd. All rights reserved.

\section{Introduction}

Food materials science has become more and more important area of food research, since there is an increasing need for understanding food stability. Water activity $\left(a_{\mathrm{w}}\right)$ or glass transition temperature $\left(T_{\mathrm{g}}\right)$ - a concept originated from the polymer science field - have been used for this purpose (Slade \& Levine, 1991). However, these concepts, do not take in account solute-solute and solutewater interactions, which influence food systems behaviour.

Despite of the in the last years, the approach of polymer science to food systems, i.e. $T_{\mathrm{g}}$, has been extensively applied in the food science and technology studies, nowadays, in order to better understand the molecular mobility concept nuclear magnetic resonance (NMR) has been presented as a powerful technique to understand and evaluate molecular mobility of semi crystalline systems, since it is able to provide information on molecular dynamics of different components in dense complex systems. As such, NMR spectroscopy has been extensively used to understand

\footnotetext{
* Corresponding author. Tel.: +351225580058.

E-mail addresses: rfernand@ibmc.up.pt (R. Fernandes), palmeida@adf.isel.pt (P.M. Almeida), gf@fct.unl.pt (G. Feio), clsilva@porto.ucp.pt (C.L.M. Silva), mquintas@porto.ucp.pt (M.A.C. Quintas).
}

the structure and dynamics of complex macromolecular systems (Domjan, Bajdik, \& Pintye-Hódi, 2009), specially biological systems (like foods) in solutions and in solid state (Claridge, 2009; Keeler, 2002; Yan, Mccarthy, Klemann, Otterburn, \& Finley, 1996). The application of this technique may be very useful in prediction of physicochemical changes namely texture, viscosity, water migration. Specifically, ${ }^{1} \mathrm{H}$ NMR has been used to investigate water dynamics and physical structures of foods through analysis of nuclear magnetisation relaxation times (Li, Kerr, Toledo, \& Carpenter, 2000). In these measurements the samples are submitted to a static magnetic field and the protons are excited by means of a radiofrequency pulse. The analysis of the signal emitted while the sample returns to equilibrium (FID) allows determining the spin-lattice $\left(T_{1}\right)$ and spin-spin $\left(T_{2}\right)$ relaxation. This later variable is related with the mobility of the protons in the samples matrix. This methodology has been applied in complex food systems such as crackers (Yan et al., 1996), wheat starch (Choi \& Kerr, 2003), chicken meat (Li et al., 2000), carrots (Rutledge, 2001) or even a model bread crust (Nieuwenhuijzen et al., 2010).

The stability of food "matrix" (system) depends strongly on its molecular mobility (as was discussed above) but also on its microstructure. Foods are highly structured and heterogeneous materials composed of architectural elements. The types of such structural 
units and their interactions are decisive in the food stability, since they influence water/solute interactions and hence the water availability to participate in degradation reactions. Microscopy techniques have been widely used in foods to study their architecture and microstructure (Aguilera, Stanley, \& Bakerc, 2000). Transmission electron microscopy (TEM) specifically, visualises the internal structure of food samples (Kaláb, Allan-Wojm, \& Miller, 1995), helping to clarify biological systems dynamics.

Edible films have been studied for a long time for their potential to improve shelf-life and safety of food products (Aider, 2010; Epure, Griffon, Pollet, \& Avérous, 2011). These systems are partially crystalline/partially amorphous, easily reproducible materials and are thus very interesting food model systems to molecular mobility studies and microstructure studies. Edible films are generally prepared by evaporation of a film forming solution composed of a natural polymer, a plasticiser and water. Depending on the evaporation method and on the viscosity and molecular entanglement of the film forming solutions the obtained film may have a different composition and/or microstructure.

The addition of low molecular weight plasticisers to amorphous biopolymers increases the matrix free volume and the molecular mobility, in an effect similar to increasing temperature (Lazaridou \& Biliaderis, 2002; Lefebvre \& Escaig, 1993). These additives reduce the tension of deformation, hardness, density, viscosity and electrostatic charge of a polymer, at the same time increase chain flexibility, resistance and dielectric constant (Ferry, 1980). Plasticisers modify the matrix second-order interactions of materials (which are responsible for crystalline structures of polymeric materials), without altering their fundamental chemical character. This modification is achieved by forming weak second-order or covalent bonds with the polymer. Plasticisers can also migrate in the polymer leading to material recrystallisation and a loss of elasticity (Domjan et al., 2009).

Plasticisers can also affect water retention capacity (Lefebvre \& Escaig, 1993). Water, considered a plasticiser, is also one of the most important solvent medium in biological systems. (Matveeva, Grinberga, \& Tolstoguzov, 2000). It greatly affects the mobility of biopolymers components and is considered as an abundant and very effective solvent/plasticiser for hydrophilic materials (Lazaridou \& Biliaderis, 2002). On a molecular level, water plasticisation of a polymer leads to increased free volume, decreased local viscosity an increased back-bone chain mobility (Slade \& Levine, 1991).

Chitosan, a polysaccharide composed mainly of $(1 \rightarrow 4)$ linked residues of $N$-acetyl $\beta$-D-glucosamine and $(1 \rightarrow 4) \beta$-D-glucosamine (Arzate-Vázqueza et al., 2012; Ostrowska-Czubenko \& Gierszewska-Drużyńska, 2009; Prashanth \& Tharanathan, 2007; Rinaudo, 2006; Yang, Liu, Wu, \& Li, 2010), is a pseudonatural cationic polymer widely used in edible films studies. Structurally, chitosan is a semicrystalline biopolymer having a huge potential for chemical and mechanical modifications to create novel properties, functions and applications in different areas (Bangyekan, Aht-Ong, \& Srikulkit, 2006; Pillai, Paul, \& Sharma, 2009; Rinaudo, 2006).

Chitosan crystal structure is stabilised by intramolecular and intermolecular $\mathrm{H}$-bonds, with the acetamide groups playing the major role in the formation of second-order bonds between adjacent chains (Okuyama, Noguchi, \& Miyazawa, 1997), making the chitosan structure on a film very dependent on the type and quantity of plasticisers used.

The objective of this study was to understand the relationship between the composition of film forming solutions and the properties (composition and microstructure) of the obtained glycerol plasticised chitosan films, evaluating the role of water and glycerol as plasticisant agents, and the effect of its concentrations in the systems performance. Also, we aimed at analysing the molecular mobility of such studies, in order to recognise its properties and improve its suitability as models for more complex food systems.
For that purpose, film forming solutions of different polymer/plasticisant concentrations were prepared and the obtained films characterised in terms of composition, molecular mobility and microstructure.

\section{Materials and methods}

\subsection{Chitosan films preparation}

Film forming solutions were prepared by dissolving different chitosan (90\% deacetylation, Aqua Premier Co., Thailand) concentrations ( $1 \%, 2 \%$ and $3 \% \mathrm{w} / \mathrm{v}$ ) in a $1 \%$ lactic acid (Acros Organics, Belgium) with three different levels of plasticising agent (10\%, 50\% and $90 \% \mathrm{~V} / \mathrm{W}$ ), glycerol (Panreac, Spain). A constant amount $(300 \mathrm{ml}$ ) of the chitosan solutions was casted in $32 \times 40 \mathrm{~cm}$ plates and dried in an incubator at $40{ }^{\circ} \mathrm{C}$, during 3 days. Films were stored at $22{ }^{\circ} \mathrm{C}$ and $53 \% \mathrm{RH}$, until equilibrium was reached prior to any characterisation.

\subsection{Characterisation of the obtained films}

\subsubsection{Chemical composition}

The final composition in chitosan and glycerol of the obtained films was determined. Chitosan concentration was estimated using a spectrophotometric method (Muzzarelli, 1998). Briefly, chitosan films were dissolved in $100 \mathrm{ml}$ of lactic acid solution $4 \%$. Cibacron brilliant red 3B-A from Sigma (Milano, Italy) was used as dye. A solution of dye was prepared by dissolving $150 \mathrm{mg}$ of the powder in ultra-pure water, using a $100 \mathrm{ml}$ volumetric flask. Aliquots of the dye solution, $5 \mathrm{ml}$, were made up to $100 \mathrm{ml}$ with $0.1 \mathrm{M}$ glycine hydrochloride buffer. Spectrophotometric measurements were done at room temperature and $575 \mathrm{~nm}$, with a UV - 1601; Shimadzu Co., Kyoto; Japan.

Glycerol concentration in films was determined using a quantitative enzymatic determination (Free Glycerol Determination Kit, from Sigma, Milano, Italy). Spectrophotometric measurements were done at room temperature and $540 \mathrm{~nm}$ using the same spectrophotometer.

\subsubsection{Thickness}

The thickness of the produced films was measured using a digital micrometre (Mitutoyo, Japan). From each sample (true replicates), a minimum of eight stripes $(15 \times 170 \mathrm{~mm})$ were cut and, at least two readings were randomly taken at different positions.

\subsubsection{Water activity}

Measurements were performed with a dew point hygrometer (Aqualab - Series 3, Decagon Devices Inc., USA.), at $23 \pm 1^{\circ} \mathrm{C}$. The sensitivity of the equipment was 0.001 . Calibration was carried out before experiments with distilled water and saturated saline solutions. Water activity value of each sample (true replicates) was the average of nine readings.

\subsubsection{Nuclear magnetic relaxation}

A Bruker AVANCE III solid state spectrometer $(300 \mathrm{MHz})$ was used to determine the samples nuclear transverse relaxation time, or spin-spin, $T_{2}$, of the protons.

These values were obtained from the exponential or bi-exponential echoes envelope of a series of Carr-Purcell-Meiboom-Gill (CPMG) multi-echo pulse sequence, which circumvents the field and sample heterogeneities and give access to the intrinsic $T_{2}$ of the protons, while the Free Induction Decay (FID) obtained from a single pulse just gives a $T_{2}^{*}$ determined mainly by the field nonuniformity in the heterogeneous film sample contained in the NMR 5 mm tubes. 
The analysis of the CPMG echoes envelope showed that the relaxation of the protons in chitosan/glycerol films follow a biexponential function. Both $t_{2}$ values were obtained by a non-linear least-square fit of the envelope data ( $T_{2}$ water and $T_{2}$ glycerol), of the function:

$A=A_{1} e^{-\frac{t}{T_{2 \text { water }}}}+A_{2} e^{-\frac{t}{T_{2 \text { glycerol }}}}$

The first point of the multi-echo acquisition pattern was normalised to unity; therefore $A_{1}$ and $A_{2}$ correspond to the respective populations' percentage.

\subsubsection{Microstructure}

Transmission electron microscope analyses were done according to a methodology described in literature (Tapia-Blácido, Do Amaral Sobral, \& Menegalli, 2011). Small pieces of films were prepared by fixation in $20 \mathrm{ml} / \mathrm{L}$ glutaraldehyde and post-fixed in $20 \mathrm{~g} /$ L OsO4. Samples were dehydrated for $15 \mathrm{~min}$ in an ethanol series $(30,50,70,90 / 100 \mathrm{ml})$, three times for $15 \mathrm{~min}$ at $99.5 / 100 \mathrm{ml}$, and twice for $20 \mathrm{~min}$ in propylene oxide. The samples were then embedded in increasing concentrations of propylene oxide: resin $(2: 1,1: 1$, and $1: 2)$ for $1 \mathrm{~h}$, and for $48 \mathrm{~h}$ in Epon 812 resin. The polymerisation of the resin subsequently proceeded at $60^{\circ} \mathrm{C}$ for $24 \mathrm{~h}$.

Ultrathin sections (40-60 nm thickness) were prepared on a Reichert SUPERNOVA LEICA Ultramicrotome (Germany) using diamond knives (DDK, Wilmington, DE, USA). The sections were mounted on 300 mesh nickel grids, and examined under a JEOL JEM 1400 TEM (Tokyo, Japan). Images were digitally recorded using a Gatan SC 1000 ORIUS CCD camera (Warrendale, PA, USA).

\section{Results and discussion}

\subsection{Composition of the films}

Drying is one of the critical processes in film preparation, since during this process, the polymer and/or plasticiser concentrations may change (Wong, Altınkaya, \& Mallapragada, 2004). In this study, the drying process was kept constant in all films, but film forming solutions with different compositions were tested.

The composition and thickness of the films obtained after drying are shown in Table 1 . It can be observed that the chitosan concentration in the obtained films do not correlate with the chitosan content of the solutions used for preparing each film. For example, films prepared using a $1 \%(\mathrm{w} / \mathrm{w})$ chitosan FFS present different final polymer content. However, it is possible to find a relationship between the glycerol added to the film forming solution and the composition of the obtained film: for solutions with constant polymer concentration, when the amount of plasticiser in the solution increases, the chitosan content in the film decreases. Moreover, it is possible to observe that polymer concentration in the solution is correlated with the thickness of the obtained films (Table 1), i.e. increasing the content of chitosan in the solution will originate thicker films.

These results suggest that films composition is governed by the amount of plasticiser in the film forming solutions, while the amount of polymer added will influence the structural rearrangement of the film. i.e. with our experiment design we obtained films with similar composition (chitosan, glycerol and water $\mathrm{mg} / \mathrm{g}$ of film) with thickness varying circa 5-fold. This may indicate that chitosan and plasticising molecules are arranged in different structures in the matrix, e.g. different crystal size and quantities and/or types. This hypothesis is supported by the fact that, these films (of same composition but different thickness) presented different crystallinity (evaluated by the change in enthalpy (results not shown) and by TEM observation (see discussion below). The phenomenon responsible for this observation is not clear. However, we can observe that the solutions used to prepare these films had all the same chitosan/glycerol ratio but different chitosan/total plasticisant (i.e. glycerol $1+$ water) ratio. This seems to indicate that this ratio is the one actually responsible for the type of bonds and interactions formed during the drying process.

\subsection{Molecular mobility}

Molecular mechanisms that control functionality in polymeric films are still poorly understood, particularly in chitosan films. Studies on the mobility of the different components at molecular level in the films matrix may help to explain structural phenomenon and simultaneously the effect of plasticiser addition.

An example obtained from NMR measurements is shown in Fig. 1. Results show that the relaxation of protons in chitosan/glycerol films follows a two components bi-exponential function, indicating the existence of two different populations with distinct relaxation behaviour (Hills, Cano, \& Belton, 1991). The two relaxation times determined in each film were assigned to water and glycerol, as they were the only components with a proton with the capacity to move. Considering the relative size of the water and glycerol molecules, the higher relaxation time, corresponding to a more mobile molecule, was assigned to the water proton and the lower relaxation times to the glycerol proton. No relaxation attributable to polymer mobility was observed in the obtained spectra even if, the plasticisers in the system are expected to soften the rigid structure of the chitosan polymeric chain (Domjan et al., 2009).

As discussed above, plasticisers are responsible for modifications in biopolymers physical properties (Hills et al., 1991). Water also acts has a plasticiser, since, water molecules can create

Table 1

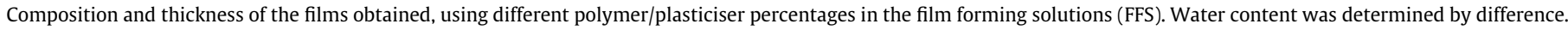

\begin{tabular}{|c|c|c|c|c|c|c|c|c|}
\hline \multicolumn{5}{|l|}{ FFS } & \multicolumn{4}{|l|}{ Films } \\
\hline Samples & Chit (g) & Gly (g) & Ratio (Chit/Gly) & $\begin{array}{l}\text { Chitosan } \\
\text { content (mg/g film) }\end{array}$ & $\begin{array}{l}\text { Glycerol } \\
\text { content }(\mathrm{mg} / \mathrm{g} \text { film })\end{array}$ & Ratio (Chit/Gly) & $\begin{array}{l}\text { Water content } \\
(\mathrm{mg} / \mathrm{g} \text { film })\end{array}$ & Thickness $^{\mathrm{a}}(\mathrm{mm})$ \\
\hline $1 \% 10 \%$ & 5 & 0.63 & 7.94 & $388.13 \pm 16.885^{a}$ & $11.47 \pm 0.019^{\mathrm{a}}$ & 33.84 & 600.40 & 0.0642 \\
\hline $1 \% 50 \%$ & 5 & 3.15 & 1.59 & $196.13 \pm 34.261^{\mathrm{a}}$ & $31.68 \pm 0.143^{\mathrm{a}}$ & 6.19 & 810.17 & 0.0556 \\
\hline $1 \% 90 \%$ & 5 & 5.67 & 0.88 & $158.15 \pm 21.535^{a}$ & $47.90 \pm 0.058^{\mathrm{a}}$ & 3.30 & 755.97 & 0.0605 \\
\hline $2 \% 10 \%$ & 10 & 1.26 & 7.94 & $356.88 \pm 23.494^{a}$ & $13.47 \pm 0.027^{\mathrm{a}}$ & 26.49 & 629.65 & 0.1344 \\
\hline $2 \% 50 \%$ & 10 & 6.31 & 1.58 & $171.85 \pm 39.978^{a}$ & $30.04 \pm 0.119^{a}$ & 5.72 & 798.11 & 0.1357 \\
\hline $2 \% 90 \%$ & 10 & 11.40 & 0.88 & $164.43 \pm 9.240^{\mathrm{a}}$ & $36.72 \pm 0.068^{\mathrm{a}}$ & 4.20 & 798.85 & 0.1527 \\
\hline $3 \% 10 \%$ & 15 & 1.89 & 7.94 & $388.14 \pm 20.396^{a}$ & $14.72 \pm 0.050^{\mathrm{a}}$ & 26.37 & 597.14 & 0.2844 \\
\hline $3 \% 50 \%$ & 15 & 9.46 & 1.59 & $181.63 \pm 30.075^{a}$ & $46.18 \pm 0.039^{a}$ & 3.93 & 772.19 & 0.2348 \\
\hline $3 \% 90 \%$ & 15 & 17.10 & 0.88 & $195.71 \pm 37.529^{a}$ & $53.36 \pm 0.083^{\mathrm{a}}$ & 3.67 & 750.93 & 0.2452 \\
\hline
\end{tabular}

a $\pm 95 \%$ confidence error. 


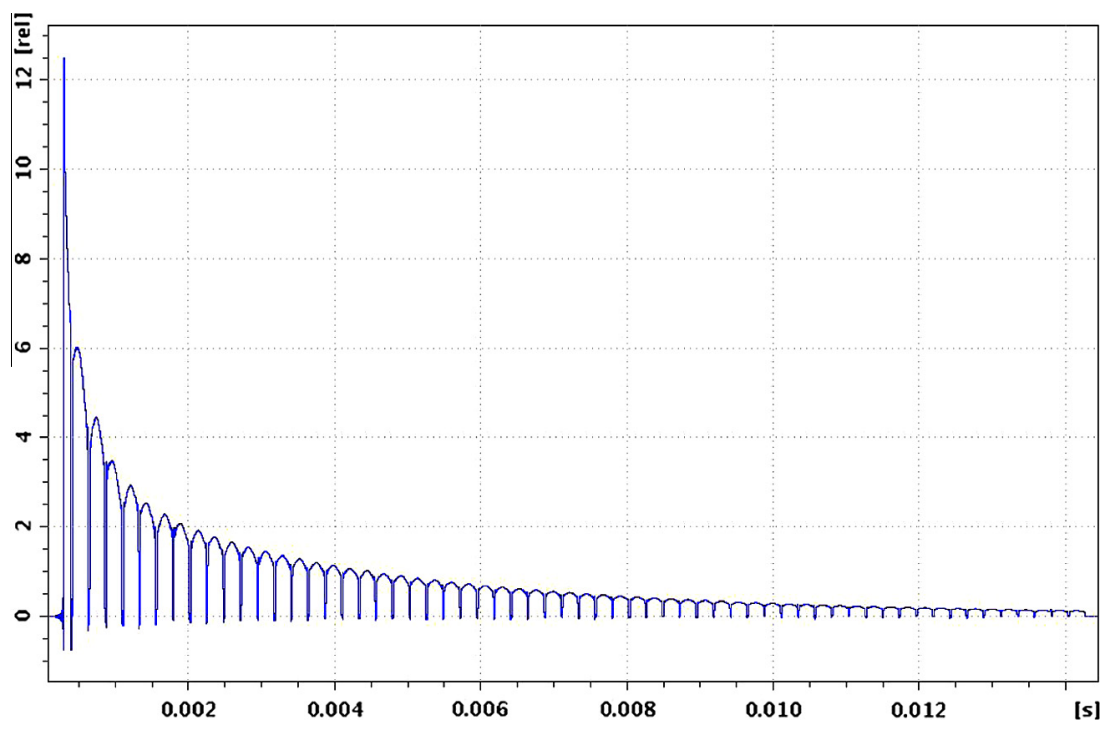

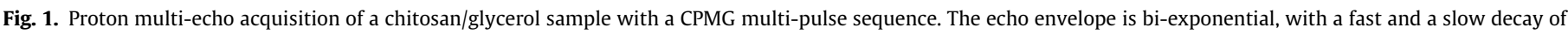
the transverse nuclear magnetism.

hydrogen bonds with the polymeric chains present in the system, thus increasing the macromolecular system free volume and contributing to a more flexible polymeric chain. In this study, the molecular mobility in the films was evaluated using nuclear magnetic resonance techniques (NMR) to determine relaxation time of the molecules present in the system.

Fig. 2a shows the transversal relaxation time $T_{2}$ of the water molecules against the composition of the different films. It is possible to observe that water relaxation time $\left(T_{2 \text { water }}\right)$, does not seem to be dependent on the chitosan content in the films, but increases with increasing glycerol content. This is due to the plasticiser effect, which reduces the intermolecular forces and increases the overall mobility in the matrix (Srinivasa, Ramesh, \& Tharanathan, 2007). On Fig. 2b, it is possible to observe that water mobility decreases with increasing chitosan/glycerol ratio in the film. A similar effect was observed in starch/glycerol films (Godbillot, Dole, Joly, Rogé, \& Mathlouthi, 2006). In this study, the authors postulate that the number of available binding sites in the polymer chain will be

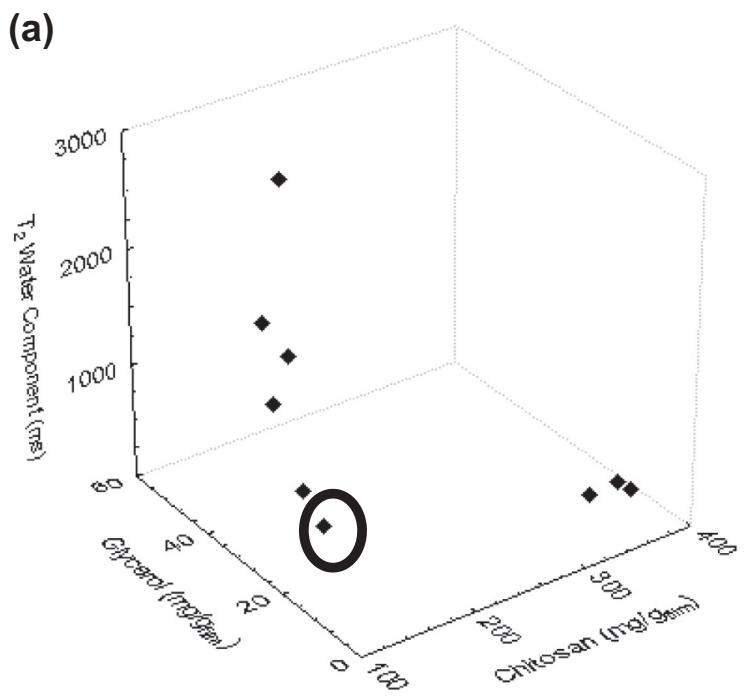

"preferentially" occupied with glycerol and water will only occupy polymer binding sites in the case no sufficient glycerol molecules were available. Our results for a chitosan/glycerol system follow the same observation: when the film glycerol content is not sufficient to occupy all the free sites on the polymer chain, the water molecules present a decreased relaxation time, indicating water is bound to chitosan. When the amount of plasticiser molecules increase, the polymer binding sites get more and more occupied by the glycerol molecules, leaving the water free to move in the chitosan matrix.

There was an exception to this behaviour: films produced with $2 \%$ of chitosan and $50 \%$ of glycerol (marked on Fig. 2 with a circle), presented a much lower relaxation time than the one observed in films with similar composition and a deviate behaviour regarding the expected relaxation time for the determined ratio Chit/Gly. This phenomenon has been reported in literature has the antiplasticisation phenomenon (Lourdin, Bizot, \& Colonna, 1997) attributable to a strong interaction occurring between the polymer and

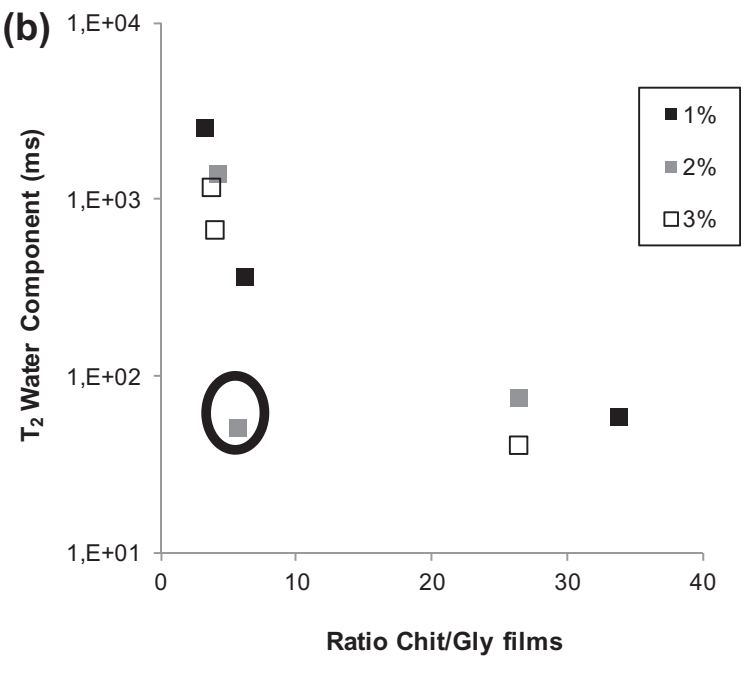

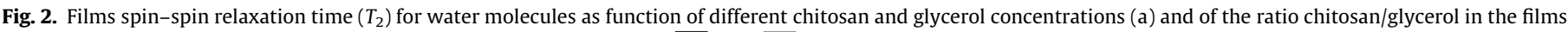
(b). Results grouped by films of same final thickness: produced with $1 \%(\square), 2 \%($ ) and $3 \%(\square)$ chitosan in the film forming solutions. 

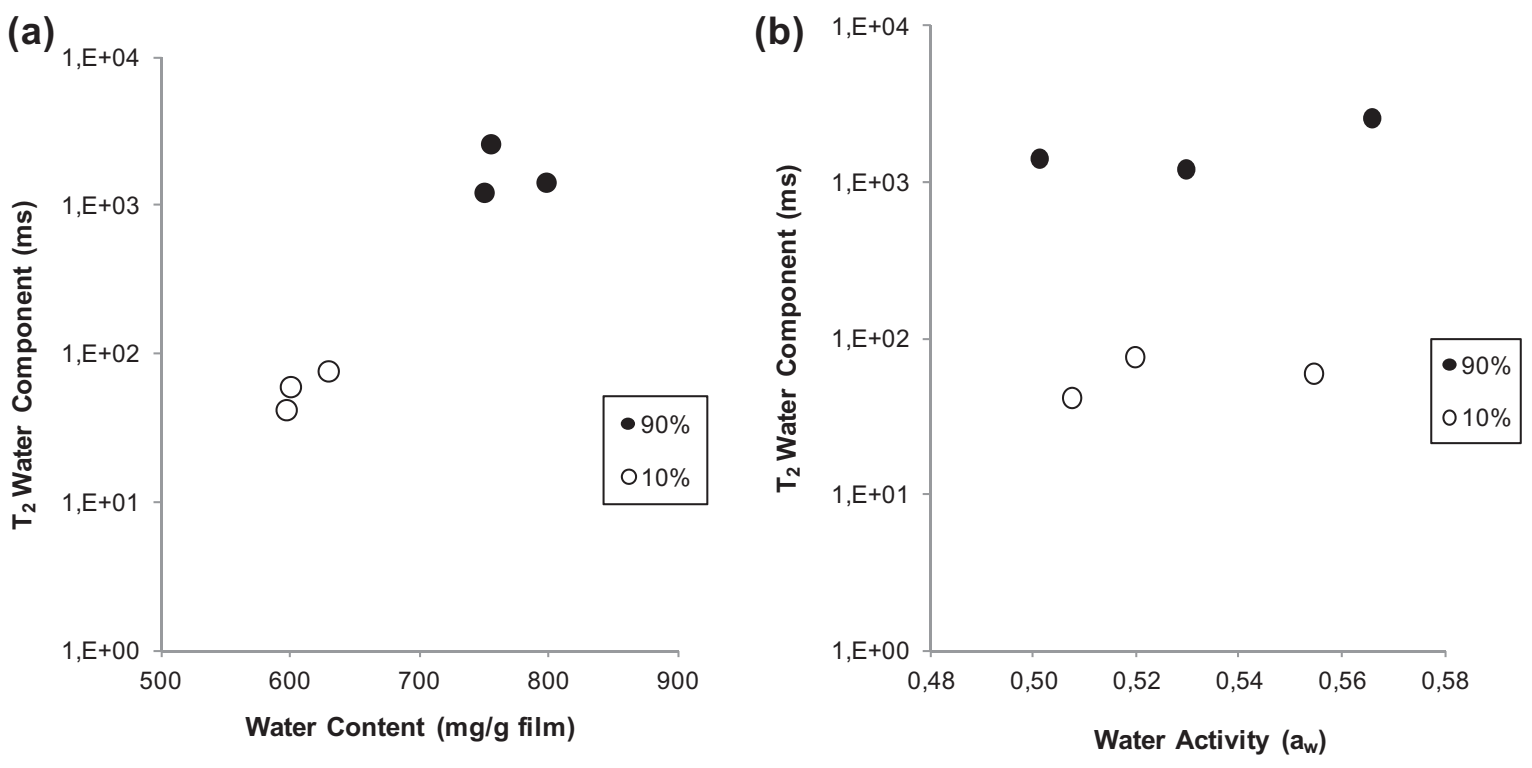

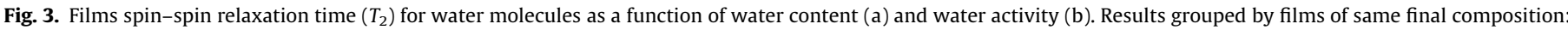

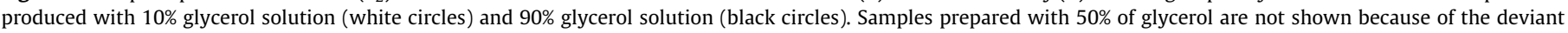
behaviour (antiplasticisation), which impairs the data analysis.

the plasticiser, producing a "cross-linker" effect, which decreases the free volume and the molecular mobility (Lourdin et al., 1997).

In order to better evaluate the role of polymer, plasticiser and/ or water binding and interactions, we compared the results of water mobility with the films water content and water activity $\left(a_{\mathrm{w}}\right)$ (Fig. 3). Fig. 3a shows the relationship between water relaxation time and water content of the films. The water molecular mobility increases with water content, which in turn is higher for samples with higher glycerol content. These results confirm the above discussed preferred affinity between chitosan and glycerol: i.e. the water molecules are free in the matrix to move because the polymer binding sites are occupied with glycerol (Godbillot et al., 2006).

Literature reports that water in biopolymer systems can be present in three different states: free in the bulk, at the surface and bound (Hills, Manning, Ridge, \& Brocklehurst, 1996). Other authors have indicated that water activity is the result of the bulk and surface water (Mathlouthi, 2001).Since water bounded to the polymeric chain has no mobility, from our results, it is clear that the relaxation time measured refers to the water in the bulk: in films of the same composition (same total water content) the increase in water activity (water at the surface and in the bulk) does not reflect on water mobility (Fig. 3b). This observation may be of great value for studies on degradation reactions in food systems and contribute for understanding differences in the stability of foods with same water activity.

In respect to the mobility of glycerol, different tendencies were observed (Fig. 4). Glycerol relaxation time $\left(T_{2}\right)$, decreased with increasing of chitosan concentrations, especially for the higher concentrations, again suggesting that glycerol may be closely bond to the chitosan chain network (Fig. 4a). This is in accordance with the results above. Again, it is possible to observe the antiplasticisation
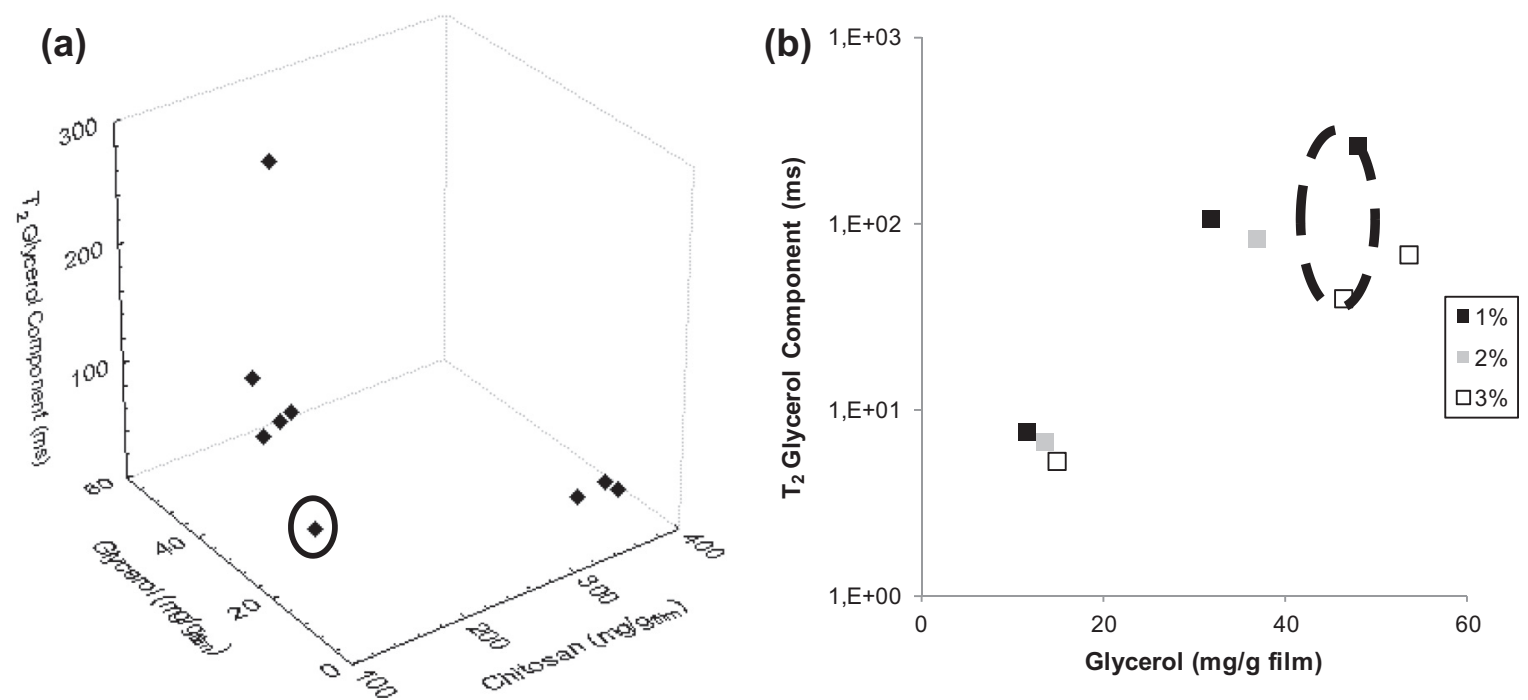

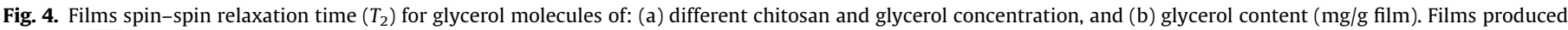
with $1 \%(\square), 2 \%(\square)$ and $3 \%(\square)$ chitosan in the FFS, each group corresponding to thickness of the obtained film. Again, antiplasticised samples are not shown. 


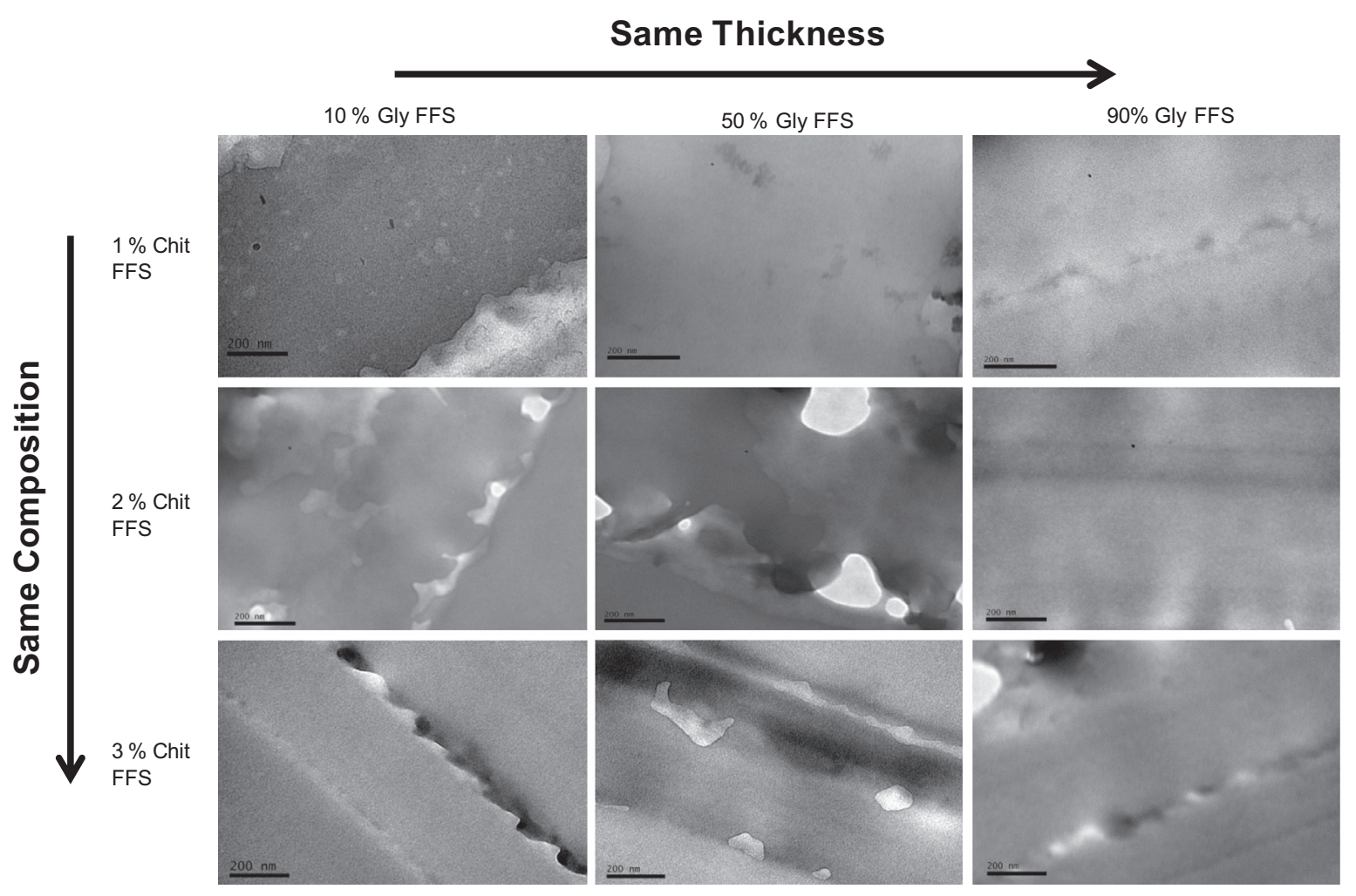

Fig. 5. TEM micrographs of films produced with different polymer/plasticiser concentrations.

phenomenon in the films produced with $2 \%$ of chitosan and $50 \%$ of glycerol (also marked with a circle).

In Fig. $4 \mathrm{~b}$ it is possible to observe the relationship between glycerol mobility and glycerol content of films, grouped by films of similar thickness. It shows that for films with the same thickness, glycerol mobility increases with increasing glycerol content. However, comparing films with approximately the same amount of glycerol, relaxation time decreases with increasing thickness of the film (see for example the data points highlighted with a dashed circle). At a first glance these results are unexpected: the films present approximately the same amount of chitosan, which would imply that an increase in thickness means an increase of the free volume in the matrix. However, the thicker films are produced with less water molecules per chitosan molecules in the film forming solution and thus it is possible that during the drying process more glycerol molecules bind to the polymeric chain - hence glycerol is not free to move in the matrix of the obtained film. This result supports our previous hypothesis that the ratio polymer/total plasticisant in the film forming solutions is critical for the type of bonds formed during the drying of the films.

\subsection{Microstructure}

As discussed above, in this study we obtained films with similar composition and significantly different thickness (Table 1 ), which may indicate differences in the films structure. To investigate such possibility, the films were observed using transmission electron microscopy (TEM). This microscopy technique allows characterising the interior of the films since the electron beam is transmitted through the sample, allowing specific observation of structures in the sample (Andreuccetti, Carvalho, \& Grosso, 2009; Denavi et al., 2009; Tapia-Blácido et al., 2011).

Chitosan films transmission electron images are presented in Fig. 5, which shows the films semicrystalline structure. The black aggregates represent the crystalline component whereas the homogeneous crowd correspond to the amorphous constituent of the sample. As above, samples images are grouped by films with similar composition and films with similar thickness.

Images show that the films with similar thickness present similar structures, with visible crystals decreasing with decreasing chitosan content: i.e. films produced with $1 \%$ Chitosan FFS have clearly evident crystals, scattered in matrix, showing a heterogeneous feature. As the chitosan content decreases, crystals are anymore observed in the obtained images. However, this may not correspond to an absence of crystals in the samples: the crystals may be smaller than the resolution of the equipment.

Furthermore, films with the same thickness were prepared by film forming solutions with the same chitosan content (see Table 1). This confirms the above hypothesis that it is the chitosan-water ratio in the film forming solutions that define the quantity/type of structures formed in the film. These structures are important because they may interfere with transport phenomena in the film and how they are formed can be important information for developing functional films. For films with similar composition, there is no apparent relationship with the structures visible in the image, which may be an indication that film composition may not be related with the functionality at macroscopic level (Vargas, Perdones, Chiralt, Cháfer, \& González-Martínez, 2011).

The results of the microstructure observations also confirm the discussions above on the polymer-plasticiser-water interactions and their effect on the molecular mobility: the films with more visible crystals correspond to the films with higher water relaxation times (higher free volume) - i.e. in the films where chitosan binding sites are "occupied" by polymer-polymer interactions in the crystalline lattice the water and glycerol molecules are free to move in the matrix. 


\section{Conclusions}

Results in this study demonstrate that glycerol quantities used in film forming solutions are responsible for chitosan concentration obtained in films and, consequently for films composition; while film forming solutions polymer/total plasticiser ratio determined the thickness (and thus structure) of the films and these conclusions were confirmed by TEM. These results can be useful for development of edible films of improved functionality.

Results on molecular mobility contributed to the understanding of the films molecular rearrangement. NMR measurements showed two different behaviours for the two components analysed, water and glycerol: while glycerol is mainly bounded to the chitosan chain network, the water present in the system is predominantly free from the polymeric chain. However, it was possible to infer that for lower glycerol concentrations, free chitosan binding sites can also be occupied by water molecules.

Water content and water activity measurements also allowed concluding that not only the water content affects the water mobility, but also structural differences in the film may influence the water relaxation time. Also it was possible to observe that water mobility relates to the water in the bulk and thus complements information on water activity of a system.

\section{Acknowledgements}

Authors Joana F. Fundo and Mafalda A.C. Quintas acknowledge Fundacão para a Ciência e a Tecnologia (grants SFRH/BD/62176/ 2009 and SFRH/BPD/41715/2007, respectively), and Portuguese NMR Network.

\section{References}

Aguilera, J. M., Stanley, D. W., \& Bakerc, K. W. (2000). New dimensions in microstructure of food products. Trends in Food Science and Technology, 11(3-9).

Aider, M. (2010). Chitosan application for active bio-based films production and potential in the food industry: Review. LWT - Food Science and Technology, 43, $837-842$.

Andreuccetti, C., Carvalho, R. A., \& Grosso, C. R. F. (2009). Effect of hydrophobic plasticizers on functional properties of gelatin-based films. Food Research International, 42(8), 1113-1121.

Arzate-Vázqueza, I., Chanona-Péreza, J. J., Calderón-Domíngueza, G., Terres-Rojasc, E., Garibay-Feblesc, V., Martínez-Rivasb, A., et al. (2012). Microstructural characterization of chitosan and alginate films by microscopy techniques and texture image analysis. Carbohydrate Polymers, 87(1), 289-299.

Bangyekan, C., Aht-Ong, D., \& Srikulkit, K. (2006). Preparation and properties evaluation of chitosan-coated cassava starch films. Carbohydrate Polymers, 63, 61-67.

Choi, S.-G., \& Kerr, W. L. (2003). ${ }^{1}$ H NMR studies of molecular mobility in wheat starch. Food Research International, 36, 341-348.

Claridge, T. D. W. (Ed.). (2009). High-resolution NMR techniques in organic chemistry. Elsevier.

Denavi, G., Tapia-Blácido, D. R., Añón, M. C., Sobral, P. J. A., Mauri, A. N., \& Menegalli, F. C. (2009). Effects of drying conditions on some physical properties of soy protein films. Journal of Food Engineering, 90(3), 341-349.

Domjan, A., Bajdik, J., \& Pintye-Hódi, K. (2009). Understanding of the plasticizing effects of glycerol and PEG 400 on chitosan films using solid-state NMR spectroscopy. Macromolecules, 42, 4667-4673.

Epure, V., Griffon, M., Pollet, E., \& Avérous, L. (2011). Structure and properties of glycerol-plasticized chitosan obtained by mechanical kneading. Carbohydrate Polymers, 83, 947-952.
Ferry, J. D. (1980). Viscoelastic properties of polymers. New York: John Wiley \& Sons, Inc.

Godbillot, L., Dole, P., Joly, C., Rogé, B., \& Mathlouthi, M. (2006). Analysis of water binding in starch plasticized films. Food Chemistry, 96, 380-386.

Hills, B. P., Cano, C., \& Belton, P. S. (1991). Proton NMR relaxation studies of aqueous polysaccharide systems. Macromolecules, 24, 2944-2950.

Hills, B. P., Manning, C. E., Ridge, Y., \& Brocklehurst, T. (1996). NMR water relaxation, water activity and bacterial survival in porous media. Journal of the Science of Food and Agriculture, 71(2), 185-194.

Kaláb, M., Allan-Wojm, P., \& Miller, S. S. (1995). Microscopy, and other imaging techniques in food structure analysis. Trends in Food Science and Technology, 6 $177-186$.

Keeler, J. (Ed.). (2002). Understanding NMR spectroscopy. Wiley - University of Cambridge, Department of Chemistry.

Lazaridou, A., \& Biliaderis, C. G. (2002). Thermophysical properties of chitosan, chitosan-starch and chitosan-pullulan films near the glass transition. Carbohydrate Polymers, 48, 179-190.

Lefebvre, J. M., \& Escaig, B. (1993). The role of molecular mobility in the yielding of solid polymers. Polymer, 34(3), 518-527.

Li, R., Kerr, W. L., Toledo, R. T., \& Carpenter, J. A. (2000). ${ }^{1} \mathrm{H}$ NMR studies of water in chicken breast marinated with different phosphates. Journal of Food Science, 65(4), 575-580.

Lourdin, D., Bizot, H., \& Colonna, P. (1997). “Antiplasticization” in starch-glycerol films? Journal of Applied Polymer Science, 63, 1047-1053.

Mathlouthi, M. (2001). Water content, water activity, water structure and the stability of foodstuffs. Food Control, 12(7), 409-417.

Matveeva, Y. I., Grinberga, V. Y., \& Tolstoguzov, V. B. (2000). The plasticizing effect of water on proteins, polysaccharides and their mixtures. Glassy state of biopolymers, food and seeds. Food Hydrocolloids, 14, 425-437.

Muzzarelli, R. A. A. (1998). Colorimetric determination of chitosan. Analytical Biochemistry, 260, 255-257.

Nieuwenhuijzen, N. H. V., Tromp, R. H., Mitchell, J. R., Primo-Martín, C., Hamer, R. J. \& Vliet, T. V. (2010). Relations between sensorial crispness and molecular mobility of model bread crust and its main components as measured by PTA, DSC and NMR. Food Research International, 43, 342-349.

Okuyama, K., Noguchi, K., \& Miyazawa, T. (1997). Molecular and crystal structure of hydrated chitosan. Macromolecules, 30, 5849-5855.

Ostrowska-Czubenko, J., \& Gierszewska-Drużyńska, M. (2009). Effect of ionic crosslinking on the water state in hydrogel chitosan membranes. Carbohydrate Polymers, 77, 590-598.

Pillai, C. K. S., Paul, W., \& Sharma, C. P. (2009). Chitin and chitosan polymers: Chemistry, solubility and fiber formation. Progress in Polymer Science, 34, 641-678.

Prashanth, K. V. H., \& Tharanathan, R. N. (2007). Chitin/chitosan: Modifications and their unlimited application potential - an overview. Trends in Food Science and Technology, 18, 117-131.

Rinaudo, M. (2006). Chitin and chitosan: Properties and applications. Progress Polymer Science, 31, 603-632.

Rutledge, D. N. (2001). Characterisation of water in agro-food products by time domain - NMR. Food Control, 12, 437-445.

Slade, L., \& Levine, H. (1991). Beyond water activity: Recent advances based on an alternative approach to the assessment of food quality and safety. Critical Reviews in Food Science and Nutrition, 30(2-3), 115-360.

Srinivasa, P. C., Ramesh, M. N., \& Tharanathan, R. N. (2007). Effect of plasticizers and fatty acids on mechanical and permeability characteristics of chitosan films. Food Hydrocolloids, 21, 1113-1122.

Tapia-Blácido, D. R., do Amaral Sobral, P. J., \& Menegalli, F. C. (2011). Optimization of amaranth flour films plasticized with glycerol and sorbitol by multi-response analysis. LWT - Food Science and Technology, 44, 1731-1738.

Vargas, M., Perdones, Á., Chiralt, A., Cháfer, M., \& González-Martínez, C. (2011) Effect of homogenization conditions on physicochemical properties of chitosanbased film-forming dispersions and films. Food Hydrocolloids, 25(5), 1158-1164.

Wong, S.-S., Altınkaya, S. A., \& Mallapragada, S. K. (2004). Drying of semicrystalline polymers: Mathematical modeling and experimental characterization of poly(vinyl alcohol) films. Polymer, 45, 5151-5161.

Yan, Z.-Y., Mccarthy, M. J., Klemann, L., Otterburn, M. S., \& Finley, J. (1996). NMR applications in complex food systems. Magnetic Resonance Image, 14(7/8) 979-981.

Yang, Y., Liu, C., Wu, H., \& Li, R. (2010). Preparation and characterization of films based on zirconium sulfophenyl phosphonate and chitosan. Carbohydrate Research, 345, 148-153. 